\title{
NUMERICAL COMPUTATION OF THE CUT LOCUS VIA A VARIATIONAL APPROXIMATION OF THE DISTANCE FUNCTION
}

\author{
François GÉnÉrau ${ }^{1}$, Edouard Oudet ${ }^{2, *}$ (i) and Bozhidar VelichKov ${ }^{3}$
}

\begin{abstract}
We propose a new method for the numerical computation of the cut locus of a compact submanifold of $\mathbb{R}^{3}$ without boundary. This method is based on a convex variational problem with conic constraints, with proven convergence. We illustrate the versatility of our approach by the approximation of Voronoi cells on embedded surfaces of $\mathbb{R}^{3}$.
\end{abstract}

Mathematics Subject Classification. 49J45, 35R35, 49M05, 35J25.

Received June 16, 2020. Accepted December 20, 2021.

\section{INTRODUCTION}

Let $S$ be a compact real analytic surface without boundary embedded in $\mathbb{R}^{3}$, and let $b \in S$ be any point of $S$ (that can be thought of as a base point).

Definition 1.1. The cut locus of $b$ in $S$ can be defined as the closure of the set of points $p \in S$ such that there exist at least two minimizing geodesics of $S$ between $p$ and $b$. We will denote it by $\operatorname{Cut}_{b}(S)$. Equivalently, it is also the set of points of $S \backslash\{b\}$ around which the distance function to the point $b$ - denoted by $d_{b}$ - is not smooth.

The cut locus is a fundamental object in Riemannian geometry, and it is a natural problem to try and find

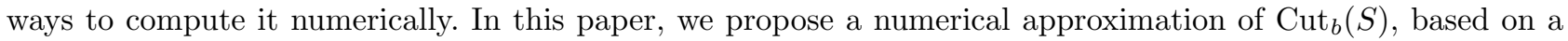
convex variational problem on $S$, with proven convergence. It is not trivial to $\operatorname{compute} \operatorname{Cut}_{b}(S)$ because it is not stable with respect to $C^{1}$-small variations of $S$. See for instance Example 2 of [1]. In particular, one can't approximate the cut locus of $S$ with the cut locus of a piecewise linear approximation of $S$.

Related works. Let us review the techniques used in the past by different authors to approximate the cut locus. We may divide them into two categories.

Geodesic approximation on parametrized surfaces. This approach was used in $[14,18]$. In [18], on genus 1 parametrized surfaces, the authors computed a degree 4 polynomial approximation of the exponential map

Keywords and phrases. Calculus of variation, cut locus, relaxation, manifold.

1 Laboratoire Jean Kuntzmann (LJK), Université Joseph Fourier, Bâtiment IMAG, 700 Avenue Centrale, 38041 Grenoble Cedex

9, France.

2 Laboratoire Jean Kuntzmann (LJK), Université Grenoble Alpes, Bâtiment IMAG, 700 Avenue Centrale, 38041 Grenoble Cedex 9, France.

3 Dipartimento di Matematica, Università di Pisa, Largo Bruno Pontecorvo, 5, 56127 Pisa, Italy.

* Corresponding author: edouard.oudet@univ-grenoble-alpes.fr

(C) The authors. Published by EDP Sciences, SMAI 2022

This is an Open Access article distributed under the terms of the Creative Commons Attribution License (https://creativecommons.org/licenses/by/4.0), which permits unrestricted use, distribution, and reproduction in any medium, provided the original work is properly cited. 
using the geodesic equation and deduced an approximation of the cut locus from there. In [14], the authors used the deformable simplicial complexes (DSC) method and finite differences techniques for geodesic computations to compute geodesic circles of increasing radius and their self-intersections, i.e. the cut locus. They applied the method to genus 1 surfaces. These papers contain no proof of convergence of the computed cut locus.

Exact geodesic computation on discretized surfaces. This approach was used in [9,13]. In [13], the authors computed the geodesics on a convex triangulated surface. They deduced an approximation of the cut locus of the triangulated surface and filtered it according to the angle formed by the geodesics meeting at a point of the approximated cut locus, to make their approximation stable. They applied the method to ellipsoids. There is no proof of convergence. In [9], the authors computed shortest curves on a graph obtained from a sufficiently dense sample of points of the surface. From there they deduced an approximation of the cut locus and filtered it according to the maximal distance (called spread) between the geodesics meeting at a point of the approximated cut locus. They proved that the set they compute converges to the cut locus (see [9], Thm. 4.1).

We may also mention [4], where the authors used some more geometric tools to compute (numerically) the cut locus of an ellipsoid or a sphere with some particular metric with singularities.

Our method. A natural approach to approximate a cut locus would be to use a fast marching method which provides an efficient way to compute distance functions on a manifold. Unfortunately, classical algorithms do not ensure any convergence result related to the gradient of the approximation. We believe that this absence of estimate makes the approximation of the cut locus by these algorithms difficult to prove. In this article we introduce a new regularized approach designed to fill this gap and to obtain a reliable localization of the cut locus. Given a large constant $m>0$, let $u_{m} \in H^{1}(S)$ be the minimizer of the following variational problem

$$
\min _{\substack{u \in H^{1}(S) \\\left|\nabla_{S} u\right| \leq 1 \\ u(b)=0}} \int_{S}\left(\left|\nabla_{S} u\right|^{2}-m u\right)
$$

where $\nabla_{S}$ denotes the gradient operator on the surface $S$. Intuitively, $u_{m}$ is a mollification away from $b$ of the distance function to the point $b$ on $S$. For $\lambda>0$ to be chosen small, we will use the set

$$
E_{m, \lambda}:=\left\{x \in S \backslash\{b\}:\left|\nabla_{S} u_{m}(x)\right|^{2} \leq 1-\frac{\lambda^{2}}{u_{m}^{2}(x)}\right\}
$$

as an approximation of $\mathrm{Cut}_{b}(S)$. See Figure 1 for an illustration of the sensitivity of $u_{m}$ and $E_{m, \lambda}$ with respect to parameters $m$ and $\lambda$.

This is justified by some theoretical results obtained in [12], which will be summarized in Sections 3 and 4 . The Sections 2-4 are devoted to explaining how we arrived at such a set $E_{m, \lambda}$. For now, let us give a bit of intuition about the different terms appearing in $E_{m, \lambda}$. When perturbing the surface $S$, we expect the same kind of instabilities as the ones observed in [3] in the case of the medial axis. Thus, two kinds of new points may appear $\operatorname{in}_{\mathrm{Cut}_{b}}(S)$ :

(1) points where some minimizing geodesics meet with an angle close to zero,

(2) points that are near the base point $b$.

Hence, to make $\operatorname{Cut}_{b}(S)$ more stable (and so more computable), we need to select points that are not too close to $b$ and such that some minimizing geodesics meet with an angle significantly larger than 0 . Intuitively, having $\left|\nabla_{S} u_{m}(x)\right|^{2} \leq 1-\alpha^{2}$ for some constant $\alpha>0$ ensures that we are selecting points $x$ where minimizing geodesics meet with an angle significantly larger than 0 , and replacing $\alpha^{2}$ by $\lambda^{2} / u_{m}^{2}$ ensures that we are selecting points that are away from $b$. Some other definitions of $E_{m, \lambda}$ would have been possible (for instance, the squares are not needed), and this form has been chosen as it corresponds to the $\lambda$-medial axis introduced in [7] (see Sect. 2).

The rest of the paper is organized as follows. In Section 2, we recall the notion of $\lambda$-medial axis that was introduced in [7] and summarize some of its properties. In Section 3, following the strategy of the $\lambda$-medial axis, 

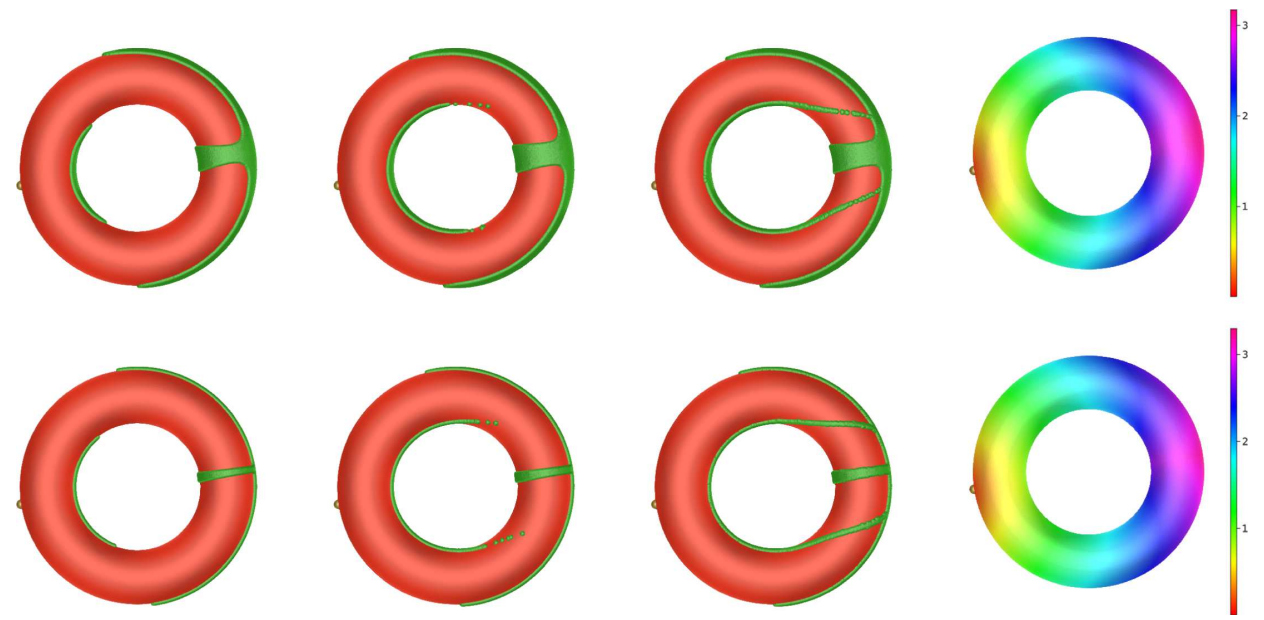

Figure 1. Approximation of the sets $E_{m, \lambda}$ (green color) for $m=10$ (first row) and $m=50$ (second row) for three different values of $\lambda$. The first three columns correspond to values of $\lambda=0.6,0.2$ and 0.06 respectively. The last column represents the associated solutions $u_{m}$.

we define a " $\lambda$-cut locus" $\operatorname{Cut}_{b}^{\lambda}(S)$ and show that it can be used as an approximation of the complete cut locus for $\lambda$ small enough. In Section 4, we recall the result from [12] which states that the set $E_{m, \lambda}$ defined above is a good approximation of $\operatorname{Cut}_{b}^{\lambda}(S)$ if $m$ is big enough. In Section 5 , we discretize problem (1.1) using finite elements, to find a discrete minimizer $u_{m, h}$, where $h>0$ is the step of the discretization. From this discrete minimizer $u_{m, h}$, we obtain a function $u_{m, h}^{l}$ on $S$, and we show that the set

$$
E_{m, \lambda, h}:=\left\{x \in S \backslash\{b\}:\left|\nabla_{S} u_{m, h}^{l}(x)\right|^{2} \leq 1-\frac{\lambda^{2}}{\left(u_{m, h}^{l}\right)^{2}(x)}\right\},
$$

is a good approximation of $E_{m, \lambda}$ as $h \rightarrow 0$. In Section 6, we present the results of some numerical experiments.

\section{THE $\lambda$-MEDIAL AXIS}

In this section, we recall briefly the notion of $\lambda$-medial axis introduced by Chazal and Lieutier [7]. Given an open subset $\Omega$ of $\mathbb{R}^{2}$, its medial axis $\mathcal{M}(\Omega)$ is defined as the set of points of $\Omega$ that have at least two closest points on the boundary $\partial \Omega$ of $\Omega$ :

$$
\mathcal{M}(\Omega):=\left\{x \in \Omega: \exists y, z \in \partial \Omega, y \neq z \text { and } d_{\partial \Omega}(x)=|x-y|=|x-z|\right\},
$$

where for any $x \in \Omega, d_{\partial \Omega}(x)$ is the distance from $x$ to $\partial \Omega$ :

$$
d_{\partial \Omega}(x)=\min \{|x-y|: y \in \partial \Omega\} .
$$

The medial axis $\mathcal{M}(\Omega)$ is unstable with respect to small non-smooth perturbations of the boundary of $\Omega$. To deal with this issue, in [7] Chazal and Lieutier defined the so called $\lambda$-medial axis of $\Omega$ by setting, for any $\lambda>0$,

$$
\mathcal{M}_{\lambda}(\Omega):=\{x \in \Omega: r(x) \geq \lambda\}
$$


where $r(x)$ is the radius of the smallest ball containing the set of all closest points to $x$ on $\partial \Omega$, i.e. the set $\left\{z \in \partial \Omega:|x-z|=d_{\partial \Omega}(x)\right\}$. The map $\lambda \mapsto \mathcal{M}_{\lambda}(\Omega)$ is nonincreasing, and

$$
\mathcal{M}(\Omega)=\bigcup_{\lambda>0} \mathcal{M}_{\lambda}(\Omega)
$$

It is further proved in Section 3, Theorem 2 of [7] that $\mathcal{M}_{\lambda}(\Omega)$ has the same homotopy type as $\mathcal{M}(\Omega)$, for $\lambda$ small enough. These facts justify that $\mathcal{M}_{\lambda}(\Omega)$ is a good approximation of $\mathcal{M}(\Omega)$, for $\lambda$ small enough. The crucial difference though is that $\mathcal{M}_{\lambda}(\Omega)$ is stable with respect to small variations of the boundary of $\Omega$, whereas $\mathcal{M}(\Omega)$ is not. We refer the reader to Section 4 of [7] for precise statements and proofs.

To motivate the next section, we will also use an alternative definition of the $\lambda$-medial axis. Given a point $x \in \Omega$, let $\Theta(x)$ be the center of the smallest ball containing all the closest points to $x$ on $\partial \Omega$. In Section 2.1 of [7], a vector field $\nabla d_{\partial \Omega}$ (originally denoted only by $\nabla$ ) is defined on $\Omega$ by:

$$
\nabla d_{\partial \Omega}(x):=\frac{x-\Theta(x)}{d_{\partial \Omega}(x)} .
$$

This vector field coincides with the classical gradient of $d_{\partial \Omega}$ wherever $d_{\partial \Omega}$ is differentiable, so it can be thought of as a generalized gradient of $d_{\partial \Omega}$. Moreover, we have the following relation (see Eq. (1) in Sect. 2.1 of [7]):

$$
\left|\nabla d_{\partial \Omega}(x)\right|^{2}=1-\frac{r^{2}(x)}{d_{\partial \Omega}^{2}(x)} .
$$

Therefore, we have the following equivalent definition of the $\lambda$-medial axis:

$$
\mathcal{M}_{\lambda}(\Omega)=\left\{x \in \Omega:\left|\nabla d_{\partial \Omega}(x)\right|^{2} \leq 1-\frac{\lambda^{2}}{d_{\partial \Omega}^{2}(x)}\right\} .
$$

\section{3. $\lambda$-Cut LOCUS}

We want to define a set similar to the $\lambda$-medial axis in the case of the cut $\operatorname{locus}^{\operatorname{Cut}_{b}}(S)$. To this end, we need a notion of generalized gradient for the distance function $d_{b}$. The notion of generalized gradient we use is presented in Section 1.3 of [16] in the context of Alexandrov spaces and in Section 2.2 of [12]. Here, we introduce the notion omitting the short proofs otherwise needed. First note that, as stated in Proposition 2.7 of [12], the function $d_{b}$ is locally semiconcave on $S \backslash\{b\}$, which means that for any unit speed geodesic $\gamma:[0,1] \rightarrow S \backslash\{b\}$, there exists a constant $C>0$ such that the function $t \mapsto C t^{2}-d_{b}(\gamma(t))$ is convex on $[0,1]$. From there, one gets that for any point $x \in S \backslash\{b\}$ and any direction $v \in T_{x} S, d_{b}$ admits a directional derivative

$$
\partial_{v}^{+} d_{b}(x)=\lim _{t \rightarrow 0^{+}} \frac{d_{b}\left(\exp _{x}(t v)\right)-d_{b}(x)}{t}
$$

where $\exp _{x}$ denotes the Riemannian exponential map at $x$. Furthermore, for any $x \in S \backslash\{b\}$, the map $v \mapsto$ $\partial_{v}^{+} d_{b}(x)$ admits a unique maximizer $v_{x}$ on the closed ball $\bar{B}(0,1) \subset T_{x} S$. The generalized gradient of $d_{b}$ is then defined as

$$
\nabla_{S} d_{b}(x)=\partial_{v_{x}}^{+} d_{b}(x) v_{x}
$$

What is more, we have the following formula

$$
\left|\nabla_{S} d_{b}(x)\right|=\max \left(0, \sup _{v \in T_{x} S,|v|=1} \partial_{v}^{+} d_{b}(x)\right) .
$$

See Lemma 3.3 for a geometric interpretation of the generalized gradient. 
Analogously to (2.2), for $\lambda>0$, we define the $\lambda$-cut locus as

$$
\operatorname{Cut}_{b}^{\lambda}(S):=\left\{x \in S \backslash\{b\}:\left|\nabla_{S} d_{b}(x)\right|^{2} \leq 1-\frac{\lambda^{2}}{d_{b}^{2}(x)}\right\} .
$$

We have the following proposition from Proposition 2.9 of [12].

Proposition 3.1. The map $\lambda \mapsto \operatorname{Cut}_{b}^{\lambda}(S)$ is nonincreasing, and

$$
\operatorname{Cut}_{b}(S)=\overline{\bigcup_{\lambda>0} \operatorname{Cut}_{b}^{\lambda}(S)}
$$

In addition, the following proposition holds. We recall that $\operatorname{Cut}_{b}(S)$ is always connected (see [15] for instance).

Proposition 3.2. If $S$ is a real analytic surface, then for $\lambda>0$ small enough, one of the connected components of $\mathrm{Cut}_{b}^{\lambda}(S)$ has the same homotopy type as $\mathrm{Cut}_{b}(S)$, while the other connected components, if any, are contractible.

These two propositions justify that $\operatorname{Cut}_{b}^{\lambda}(S)$ is a good approximation of $\operatorname{Cut}_{b}(S)$, for $\lambda>0$ small enough. Before proving Proposition 3.2, we prove the following lemma.

Lemma 3.3. Let $x \in \operatorname{Cut}_{b}(S)$ be such that there exist two unit speed minimizing geodesics $\gamma_{1}, \gamma_{2}:\left[0, d_{b}(x)\right] \rightarrow S$ such that $\gamma_{i}(0)=b$ and $\gamma_{i}\left(d_{b}(x)\right)=x$. Let $\theta \in(0, \pi]$ be the angle between $\gamma_{1}$ and $\gamma_{2}$ at $x$. Then, we have

$$
\left|\nabla_{S} d_{b}(x)\right| \leq \cos (\theta / 2) .
$$

Proof. For $i=1,2$, let us set $v_{i}=-\dot{\gamma}_{i}\left(d_{b}(x)\right)$. Let us denote by $\exp _{x}$ the Riemannian exponential map at the point $x$. Let $t_{0} \in\left(0, d_{b}(x)\right)$ and $x_{i}=\exp _{x}\left(v_{i} t_{0}\right)$. Note that we have $x \notin \operatorname{Cut}_{x_{i}}(S)$, so the function $d_{x_{i}}$ is smooth at $x$, and its gradient is $-v_{i}$. Given $v \in T_{x} S$ such that $|v|=1$, using $d_{b}(x)=d_{x_{i}}(x)+d_{b}\left(x_{i}\right)$, we have

$$
\begin{aligned}
\partial_{v}^{+} d_{b}(x) & =\lim _{t \rightarrow 0^{+}} \frac{d_{b}\left(\exp _{x}(v t)\right)-d_{b}(x)}{t} \\
& \leq \lim _{t \rightarrow 0^{+}} \frac{d_{x_{i}}\left(\exp _{x}(v t)\right)+d_{b}\left(x_{i}\right)-\left(d_{x_{i}}(x)+d_{b}\left(x_{i}\right)\right)}{t} \\
& =\lim _{t \rightarrow 0^{+}} \frac{d_{x_{i}}\left(\exp _{x}(v t)\right)-d_{x_{i}}(x)}{t} \\
& =-v \cdot v_{i} .
\end{aligned}
$$

Given that the angle between $v_{1}$ and $v_{2}$ is $\theta$, there exists $i \in\{1,2\}$, such that the angle between $v$ and $v_{i}$ is at most $\pi-\theta / 2$. Thus the last inequality gives $\partial_{v}^{+} d_{b}(x) \leq \cos (\theta / 2)$. This concludes the proof.

Remark 3.4. In the previous lemma, in the case where there are exactly two minimizing geodesics arriving at $x$, one can show that the inequality is an equality.

Using Lemma 3.3, Proposition 3.2 will mainly be a consequence of Proposition 3.4 from [9], which is recalled in Proposition 3.6 below. Following [9], we will use the following terminology. Let $G$ be a finite connected graph embedded in $S$. A point $x$ of a finite connected graph $G$ is called a tree point if $x$ is a leaf of $G$ or $G \backslash\{x\}$ has a connected component whose closure is a tree. Otherwise, $x$ is called a cycle point. As shown in the proof of Proposition 3.5 from [9], the closure of the set of cycle points of a finite connected graph $G$ is a deformation retract of $G$, hence it is connected. We will also use the following lemma.

Lemma 3.5. Let $G$ be a finite connected graph. Let $\mathcal{C} \subset G$ be a closed connected set that contains all cycle points of $G$. Then $\mathcal{C}$ is a deformation retract of $G$. 
Proof. Let $T_{1}, \ldots, T_{k}$ be the connected components of $G \backslash \mathcal{C}$. As $\mathcal{C}$ contains all cycle points of $G$, for any $1 \leq i \leq k, T_{i}$ is a tree and $\overline{T_{i}} \cap \mathcal{C}$ is a singleton $\left\{x_{i}\right\}$. By contracting all $T_{i}$ to their roots $x_{i}$, we obtain that $\mathcal{C}$ is a deformation retract of $G$.

Let $x \in S$ be such that there exist two minimizing unit speed geodesics $\gamma_{1}$ and $\gamma_{2}$ from $b$ to $x$. Following [9], the spread between $\gamma_{1}$ and $\gamma_{2}$ is defined as

$$
\operatorname{spd}\left(\gamma_{1}, \gamma_{2}\right)=\sup _{t} d\left(\gamma_{1}(t), \gamma_{2}(t)\right)
$$

We recall that, as $S$ is real analytic, the cut $\operatorname{locus} \operatorname{Cut}_{b}(S)$ is a finite graph (see [15] in dimension 2 and [5] for the generalization to arbitrary dimensions). In [9], the authors proved the following:

Proposition 3.6 ([9], Prop. 3.4). Let $x \in \mathrm{Cut}_{b}(S)$. If the spread of any two minimizing unit speed geodesics $\gamma_{1}$ and $\gamma_{2}$ from $b$ to $x$ is smaller than the injectivity radius of $S$, then $x$ is a tree point of $\operatorname{Cut}_{b}(S)$.

Proof of Proposition 3.2. According to Lemma 3.3, given any $\theta>0$, if $\lambda$ has been taken small enough, then for any point $x \in \operatorname{Cut}_{b}(S) \backslash \mathrm{Cut}_{b}^{\lambda}(S)$, the angle between any two minimizing unit speed geodesics $\gamma_{1}$ and $\gamma_{2}$ from $b$ to $x$ is smaller than $\theta$ at $x$. As geodesics verify a second order differential equation, if their angle at $x$ is small, then their spread is also small. Therefore, applying Proposition 3.6, we deduce that if $\lambda$ has been taken small enough, then any point $x \in \operatorname{Cut}_{b}(S) \backslash \operatorname{Cut}_{b}^{\lambda}(S)$ is a tree point of $\operatorname{Cut}_{b}(S)$. Stated otherwise, $\operatorname{Cut}_{b}^{\lambda}(S)$ contains all cycle points of $\operatorname{Cut}_{b}(S)$. Moreover, $\operatorname{Cut}_{b}^{\lambda}(S)$ is a closed set. Indeed, this is a consequence of the semiconcavity of $d_{b}$ and the lower semicontinuity of the norm of the gradient of semiconcave functions (see [12], Prop. 7.2). Thus, $\operatorname{Cut}_{b}^{\lambda}(S)$ contains the closure of the cycle points of $\operatorname{Cut}_{b}(S)$, which is connected. In particular, there exists a connected component $\mathcal{C}$ of $\operatorname{Cut}_{b}^{\lambda}(S)$ that contains the set of the cycle points of $\operatorname{Cut}_{b}(S)$. By Lemma 3.5, $\mathcal{C}$ is a deformation retract of $\operatorname{Cut}_{b}(S)$. This completes the proof.

Therefore, we will use $\operatorname{Cut}_{b}^{\lambda}(S)$ as an approximation of $\operatorname{Cut}_{b}(S)$ for $\lambda$ small enough.

\section{Approximation with a Variational problem}

For $m>0$, recall that $u_{m}$ is the minimizer in (1.1). For $\lambda>0$, let us define the set $E_{m, \lambda}$ by

$$
E_{m, \lambda}:=\left\{x \in S \backslash\{b\}:\left|\nabla_{S} u_{m}(x)\right|^{2} \leq 1-\frac{\lambda^{2}}{u_{m}^{2}(x)}\right\}
$$

We have the following theorem (see [12], Thms. 1.1 and 1.3):

Theorem 4.1. There exists $m_{0}>0$ such that for any $m>m_{0}$, the function $u_{m}$ is locally $C^{1,1}$ on $S \backslash\{b\}$, and $u_{m}=d_{b}$ in a neighborhood of $b$. For any $m>m^{\prime}>m_{0}$,

$$
\operatorname{Cut}_{b}(S) \subset\left\{\left|\nabla_{S} u_{m}\right|<1\right\} \subset\left\{\left|\nabla_{S} u_{m^{\prime}}\right|<1\right\} .
$$

Moreover,

$$
\left\{\left|\nabla_{S} u_{m}\right|<1\right\} \underset{m \rightarrow+\infty}{\longrightarrow} \operatorname{Cut}_{b}(S) \text { in the Hausdorff sense. }
$$

Finally, for any $\varepsilon>0$,

$$
\sup _{x \in E_{m, \lambda}} d\left(x, \operatorname{Cut}_{b}^{\lambda}(S)\right) \underset{m \rightarrow+\infty}{\longrightarrow} 0, \quad \text { and } \sup _{x \in \operatorname{Cut}_{b}^{\lambda+\varepsilon}(S)} d\left(x, E_{m, \lambda}\right) \underset{m \rightarrow+\infty}{\longrightarrow} 0 .
$$

Therefore, we can use $E_{m, \lambda}$ as an approximation of $\operatorname{Cut}_{b}^{\lambda}(S)$. All in all, we will use $E_{m, \lambda}$ as an approximation of $\operatorname{Cut}_{b}(S)$.

In the following, we will always assume that we have $m>m_{0}$. 


\section{Discretization}

\subsection{Finite elements of order $r$ on a surface approximation of order $k$}

In this section we introduce a discretization framework adapted to the variational problem (1.1), based on finite elements. We follow the notations of $[8,11]$.

Let $S$ be a compact oriented smooth two-dimensional surface embedded in $\mathbb{R}^{3}$. For $x \in S$, we denote by $\nu(x)$ the oriented normal vector field on $S$. Let $d: \mathbb{R}^{3} \rightarrow \mathbb{R}$ be the signed distance function to the surface $S$ and $U_{\eta}=\left\{x \in \mathbb{R}^{3},|d(x)|<\eta\right\}$ the tubular neighborhood of $S$ of width $\eta>0$. It is well known that if $\eta$ is small enough (for instance $0<\eta<\min _{i=1,2} \frac{1}{\left|\kappa_{i}\right|} L^{\infty}(S)$ where the $\left(\kappa_{i}\right)$ stand for the extremal principal curvatures of S), then for every $x \in U_{\eta}$, there exists a unique $a(x) \in S$ such that

$$
x=a(x)+d(x) \nu(a(x))=a(x)+d(x) \nabla d(x) .
$$

We consider $S_{h}$ a triangular approximation of $S$ whose vertices lie on $S$ and whose faces are quasi-uniform and shape regular of diameter at most $h>0$. Moreover, we will assume that $\mathcal{T}_{h}$, the set of triangular faces of $S_{h}$, are contained in some tubular neighborhood $U_{\eta}$ such that the map $a$ defined by (5.1) is unique.

For $k \geq 1$ and for a triangle $T \in \mathcal{T}_{h}$, we consider the $n_{k}$ Lagrange basis functions $\Phi_{1}^{k}, \ldots \Phi_{n_{k}}^{k}$ of degree $k$ and define the discrete projection on $S_{h}$ by:

$$
a_{k}(x)=\sum_{j=1}^{n_{k}} a\left(x_{j}\right) \Phi_{j}^{k}(x)
$$

where $x_{1}, \ldots, x_{n_{k}}$ are the nodal points associated to the basis functions. Now we can define $S_{h}^{k}$ a polynomial approximation of order $k$ of $S$ associated to $\mathcal{T}_{h}$

$$
S_{h}^{k}=\left\{a_{k}(x), x \in S_{h}\right\} .
$$

Observe that by definition the image by $a$ of the nodal points are both on $S$ and on $S_{h}^{k}$. Let us now introduce the finite element spaces on $S_{h}=S_{h}^{1}$ and $S_{h}^{k}$ for $k \geq 2$. For every integer $r \geq 1$, let

$$
L_{h}^{r}=\left\{\chi \in C^{0}\left(S_{h}\right),\left.\chi\right|_{T} \in \mathbb{P}_{r}, \forall T \in \mathcal{T}_{h}\right\}
$$

where $\mathbb{P}_{r}$ is the family of polynomials of degree at most $r$. Analogously, for $k \geq 2$, let

$$
L_{h}^{r, k}=\left\{\hat{\chi} \in C^{0}\left(S_{h}^{k}\right), \hat{\chi}=\chi \circ a_{k}^{-1}, \text { for some } \chi \in L_{h}^{r}\right\} .
$$

Analogously to (1.1), we will consider the following discrete variational problem:

$$
\min _{\substack{u \in L_{h}^{r, k} \\
\mid \begin{array}{c}
\nabla_{S_{h}^{k}} u \mid \leq 1 \\
u(b)=0 \\
u(b)=0
\end{array}}} F_{h}^{k}(u)
$$

where $F_{h}^{k}(u)=\int_{S_{h}^{k}}\left(\left|\nabla_{S_{h}^{k}} u\right|^{2}-m u\right)$ and $b$ some fixed nodal point of the mesh $\mathcal{T}_{h}$.

\subsection{Convergence of the lifted minimizers}

In order to prove the convergence of our numerical approach, let us first establish that our discrete problem converges in values in the sense of Proposition 5.2. For a function $u$ defined on $S_{h}^{k}$, we introduce its lifted function $u^{l}$ defined on $S$, by the relation $u^{l}(a(x))=u(x)$.

We focus our analysis on the piecewise linear case $r=k=1$. We will use the notation $F_{h}:=F_{h}^{1}$ and $L_{h}:=L_{h}^{1}$. For every $h>0$, the convex optimization problem (5.6) has a unique solution $u_{m, h}$. 
Lemma 5.1. The differential of the projection $a: U_{\eta} \rightarrow \mathbb{R}^{3}$ onto $S$, when restricted to the tangent space of $S_{h}$, is the identity, up to order 1 in h:

$$
D a_{\left.\right|_{T S_{h}}}=I d_{\left.\right|_{T S_{h}}}^{\mid \mathbb{R}^{3}}+\mathcal{O}(h)
$$

Proof. The identity estimate on $D a$ is a direct consequence of [11] equations (4.12), (4.13), (4.10) and (4.11).

Defining $F(u)=\int_{S}\left(\left|\nabla_{S} u\right|^{2}-m u\right)$, we have the following proposition.

Proposition 5.2. Let $u_{m, h}$ be the solution of problem (5.6) for $k=r=1$. Let $L u_{m, h}^{l}:=\frac{u_{m, h}^{l}}{\max \left(\left|\nabla_{S} u_{m, h}^{l}\right|_{L^{\infty}(S)}, 1\right)}$ be the 1-Lipschitz normalization of $u_{m, h}^{l}$. Then, $L u_{m, h}^{l} \in H^{1}(S)$ and

$$
F\left(L u_{m, h}^{l}\right)=\min _{\substack{u \in H^{1}(S) \\\left|\nabla_{S} u\right| \leq 1 \\ u(b)=0}} F(u)+\mathcal{O}\left(h^{\frac{1}{2}}\right) .
$$

Proof. Step 1. Let $u_{m}$ be the solution of problem (1.1). For $\varepsilon>0$, let $w_{m, \varepsilon}: S \rightarrow \mathbb{R}$ be defined by:

$$
w_{m, \varepsilon}=\left\{\begin{array}{lll}
\frac{d_{b}(x)^{2}}{2 \varepsilon} & \text { if } & d_{b}(x) \leq \varepsilon \\
u_{m}(x)-\frac{\varepsilon}{2} & \text { if } & d_{b}(x) \geq \varepsilon .
\end{array}\right.
$$

Recall that we have $u_{m}=d_{b}$ in a neighborhood of $b$ (Thm. 4.1). Therefore, for $\varepsilon>0$ small enough, we have $u_{m}=d_{b}$ on the ball $B(b, 2 \varepsilon)$. In particular, we deduce that $w_{m, \varepsilon}$ is $C^{1}$ on $S$. As $d_{b}^{2}$ is smooth in a neighborhood of $b$, the gradient of $d_{b}^{2} / 2 \varepsilon$ is $\mathcal{O}\left(\varepsilon^{-1}\right)$-Lipschitz on $B(b, \varepsilon)$. Moreover, as $u_{m}=d_{b}$ on $B(b, 2 \varepsilon)$, the gradient of $u_{m}$ is $\mathcal{O}\left(\varepsilon^{-1}\right)$-Lipschitz on $B(b, 2 \varepsilon) \backslash B(b, \varepsilon)$. Recall that $u_{m}$ is also locally $C^{1,1}$ on $S \backslash\{b\}$ (Thm. 4.1). Therefore its gradient is $\mathcal{O}\left(\varepsilon^{-1}\right)$-Lipschitz on $S \backslash B(b, \varepsilon)$. All in all, we obtain that $w_{m, \varepsilon}$ is $C^{1,1}$ on $S$ and the Lipschitz constant of its gradient is $\mathcal{O}\left(\varepsilon^{-1}\right)$. Furthermore, as $d_{b}$ and $u_{m}$ are both 1-Lipschitz, we have $\left|\nabla_{S} w_{m, \varepsilon}\right| \leq 1$. Now for $\varepsilon>0$, consider

$$
v_{h, \varepsilon}:=\frac{I_{h} w_{m, \varepsilon}}{\left|\nabla_{S_{h}} I_{h} w_{m, \varepsilon}\right|_{L^{\infty}\left(S_{h}\right)}},
$$

where $I_{h} w_{m, \varepsilon}$ is the $\mathbb{P}^{1}$ Lagrange interpolation of $w_{m, \varepsilon}$ on $S_{h}$. Therefore, $v_{h, \varepsilon}$ is an admissible candidate in the minimization problem (5.6). For $x \in S_{h}$, observe that we have the relation $I_{h} w_{m, \varepsilon}(x)=I_{h}\left(w_{m, \varepsilon} \circ a\right)(x)$ which says that $I_{h} w_{m, \varepsilon}$ is the standard (flat) interpolation of the composed function $w_{m, \varepsilon} \circ a$. As the map $a: U_{\eta} \rightarrow S$ is smooth and the gradient of $w_{m, \varepsilon}$ is $\mathcal{O}\left(\varepsilon^{-1}\right)$-Lipschitz, we deduce that on every triangle of $S_{h}$, the gradient of $w_{m, \varepsilon} \circ a$ is $\mathcal{O}\left(\varepsilon^{-1}\right)$-Lipschitz, uniformly in $h$. By the quasi uniformity of the mesh, we obtain the uniform interpolation estimates on $S_{h}$ :

$$
I_{h} w_{m, \varepsilon}(x)=\left(w_{m, \varepsilon} \circ a\right)(x)+\mathcal{O}\left(\varepsilon^{-1} h^{2}\right)
$$

and

$$
\nabla_{S_{h}} I_{h} w_{m, \varepsilon}(x)=\nabla_{S_{h}}\left(w_{m, \varepsilon} \circ a\right)(x)+\mathcal{O}\left(\varepsilon^{-1} h\right) .
$$

With Lemma 5.1, we deduce for all $x \in S_{h}$,

$$
\nabla_{S_{h}} I_{h} w_{m, \varepsilon}(x)=\nabla_{S} w_{m, \varepsilon}(a(x))+\mathcal{O}\left(\varepsilon^{-1} h\right) .
$$


Recall that we have $\left|\nabla_{S} w_{m, \varepsilon}\right|_{L^{\infty}(S)}=1$. Therefore the last identity yields

$$
\left|\nabla_{S_{h}} I_{h} w_{m, \varepsilon}\right|_{L^{\infty}\left(S_{h, \varepsilon}\right)}=1+\mathcal{O}\left(\varepsilon^{-1} h\right) .
$$

Thus, $v_{h, \varepsilon}=I_{h} w_{m, \varepsilon}\left(1+\mathcal{O}\left(\varepsilon^{-1} h\right)\right)$, and so

$$
F_{h}\left(v_{h, \varepsilon}\right)=F_{h}\left(I_{h} w_{m, \varepsilon}\right)+\mathcal{O}\left(\varepsilon^{-1} h\right) .
$$

Applying Lemma 5.1 again, with a simple change of variable, we find that for any function $f: S \rightarrow \mathbb{R}$,

$$
\int_{S_{h}} f \circ a=\int_{S} f+\mathcal{O}(h) .
$$

Recalling (5.7) and (5.8), we obtain

$$
F_{h}\left(I_{h} w_{m, \varepsilon}\right)=F\left(w_{m, \varepsilon}\right)+\mathcal{O}\left(\varepsilon^{-1} h\right) .
$$

Furthermore, we have

$$
\int_{S}\left|w_{m, \varepsilon}-u_{m}\right| \leq \mathcal{O}(\varepsilon) \quad \text { and }\left.\int_{S}|| \nabla_{S} w_{m, \varepsilon}\right|^{2}-\left|\nabla_{S} u_{m}\right|^{2} \mid \leq \mathcal{O}\left(\varepsilon^{2}\right),
$$

so

$$
F\left(w_{m, \varepsilon}\right)=F\left(u_{m}\right)+\mathcal{O}(\varepsilon) .
$$

Combining this with (5.9) and (5.11), we find

$$
F_{h}\left(v_{h, \varepsilon}\right)=F\left(u_{m}\right)+\mathcal{O}\left(\varepsilon^{-1} h\right)+\mathcal{O}(\varepsilon) .
$$

Choosing $\varepsilon=h^{\frac{1}{2}}$, this yields

$$
\min _{\substack{u \in L_{h} \\\left|\nabla_{S_{h}} u\right| \leq 1 \\ u(b)=0}} F_{h}(u) \leq \min _{\substack{u \in H^{1}(S) \\\left|\nabla_{S} u\right| \leq 1 \\ u(b)=0}} F(w)+\mathcal{O}\left(h^{\frac{1}{2}}\right) .
$$

Step 2. Let $u_{m, h}$ be the solution of the discrete problem (5.6), $u_{m, h}^{l}:=u_{m, h} \circ\left(a_{\left.\right|_{S_{h}}}\right)^{-1}$ its lifted version on $S$, and

$$
L u_{m, h}^{l}:=\frac{u_{m, h}^{l}}{\max \left(\left|\nabla_{S} u_{m, h}^{l}\right|_{L^{\infty}(S)}, 1\right)} .
$$

Using the equation $u_{m, h}=u_{m, h}^{l} \circ a$ and Lemma 5.1 as before, we obtain that uniformly for $x \in S_{h}$,

$$
\nabla_{S} u_{m, h}^{l} \circ a(x)=\nabla_{S_{h}} u_{m, h}(x)+\mathcal{O}(h) .
$$

In particular, this implies

$$
\left|\nabla_{S} u_{m, h}^{l}\right|_{L^{\infty}(S)}=\left|\nabla_{S_{h}} u_{m, h}\right|_{L^{\infty}\left(S_{h}\right)}+\mathcal{O}(h) \leq 1+\mathcal{O}(h),
$$

and so

$$
L u_{m, h}^{l}=u_{m, h}^{l}(1+\mathcal{O}(h)) .
$$


From this identity we deduce

$$
F\left(L u_{m, h}^{l}\right)=F\left(u_{m, h}^{l}\right)+\mathcal{O}(h) .
$$

Using the estimates (5.13) and (5.10) as in step one, we find

$$
F_{h}\left(u_{m, h}\right)=F\left(u_{m, h}^{l}\right)+\mathcal{O}(h)
$$

The last two equations together yield $F\left(L u_{m, h}^{l}\right)=F_{h}\left(u_{m, h}\right)+\mathcal{O}(h)$. With (5.12), this implies

$$
\min _{\substack{u \in H^{1}(S) \\\left|\nabla_{S} u\right| \leq 1 \\ u(b)=0}} F(u) \leq F\left(L u_{m, h}^{l}\right) \leq \min _{\substack{u \in H^{1}(S) \\\left|\nabla_{S} u\right| \leq 1 \\ u(b)=0}} F(u)+\mathcal{O}\left(h^{\frac{1}{2}}\right),
$$

which concludes the proof of the proposition.

In the proof of the next proposition, we will need the following lemma.

Lemma 5.3. Let $L>0$. Let $\left(f_{h}\right)_{h>0}$ be a family of L-Lipschitz real functions on $S$ such that $\left|\nabla f_{h}\right|_{L^{1}(S)} \rightarrow 0$ as $h \rightarrow 0$ and for any $h>0, f_{h}(b)=0$. Then

$$
\left|f_{h}\right|_{L^{1}(S)} \underset{h \rightarrow 0}{\longrightarrow} 0 .
$$

Proof. Let $\varepsilon>0$ and $S_{\varepsilon}:=S \backslash\left(\operatorname{Cut}_{b}(S) \cup B(b, \varepsilon)\right)$. We will use the polar coordinates $(r, \theta)$ centered at $b$ on $S_{\varepsilon}$. In these coordinates, the surface measure on $S_{\varepsilon}$ is of the form $\mathrm{d} \mathcal{A}=A(r, \theta) \mathrm{d} r \mathrm{~d} \theta$, with

$$
A(r, \theta) \underset{r \rightarrow 0}{\sim} r, \quad \text { uniformly in } \theta .
$$

Moreover, the surface $S_{\varepsilon}$ is of the form

$$
S_{\varepsilon}=\left\{(r, \theta), \theta \in[0,2 \pi), r \in\left[\varepsilon, r_{\theta}\right)\right\},
$$

for some $r_{\theta}>0$ that depends on $\theta \in[0,2 \pi)$. As the $\left(f_{h}\right)_{h>0}$ are $L$-Lipschitz and $f_{h}(b)=0$, we have for any $\theta \in[0,2 \pi),\left|f_{h}(\varepsilon, \theta)\right| \leq L \varepsilon$. Using this inequality, we have

$$
\begin{aligned}
\int_{S_{\varepsilon}}\left|f_{h}\right| & =\int_{0}^{2 \pi} \int_{\varepsilon}^{r_{\theta}}\left|f_{h}(r, \theta)\right| A(r, \theta) \mathrm{d} r \mathrm{~d} \theta \\
& \leq \int_{0}^{2 \pi} \int_{\varepsilon}^{r_{\theta}}\left|f_{h}(r, \theta)-f_{h}(\varepsilon, \theta)\right| A(r, \theta) \mathrm{d} r \mathrm{~d} \theta+\int_{0}^{2 \pi} \int_{\varepsilon}^{r_{\theta}}\left|f_{h}(\varepsilon, \theta)\right| A(r, \theta) \mathrm{d} r \mathrm{~d} \theta \\
& \leq \int_{0}^{2 \pi} \int_{\varepsilon}^{r_{\theta}}\left|f_{h}(r, \theta)-f_{h}(\varepsilon, \theta)\right| A(r, \theta) \mathrm{d} r \mathrm{~d} \theta+\mathcal{A}(S) L \varepsilon \\
& =\int_{0}^{2 \pi} \int_{\varepsilon}^{r_{\theta}}\left|\int_{\varepsilon}^{r} \partial_{r} f_{h}(t, \theta) \mathrm{d} t\right| A(r, \theta) \mathrm{d} r \mathrm{~d} \theta+\mathcal{A}(S) L \varepsilon \\
& \leq \int_{0}^{2 \pi} \int_{\varepsilon}^{r_{\theta}} \int_{\varepsilon}^{r}\left|\nabla f_{h}(t, \theta)\right| \mathrm{d} t A(r, \theta) \mathrm{d} r \mathrm{~d} \theta+\mathcal{A}(S) L \varepsilon .
\end{aligned}
$$

From (5.15), we know that for some constants $C_{1}, C_{2}>0$, we have for any $r>0$ and $\theta \in[0,2 \pi), C_{1} r \leq A(r, \theta) \leq$ $C_{2}$. In particular, for $r \geq t \geq \varepsilon$, we get

$$
A(r, \theta) \leq C_{2} \leq C_{2} \frac{t}{\varepsilon} \leq \frac{C_{2}}{C_{1} \varepsilon} A(t, \theta) .
$$


Setting $C:=C_{2} / C_{1}$, with (5.16), we find

$$
\begin{aligned}
\int_{S_{\varepsilon}}\left|f_{h}\right| & \leq \frac{C}{\varepsilon} \int_{0}^{2 \pi} \int_{\varepsilon}^{r_{\theta}} \int_{\varepsilon}^{r}\left|\nabla f_{h}(t, \theta)\right| A(t, \theta) \mathrm{d} t \mathrm{~d} r \mathrm{~d} \theta+\mathcal{A}(S) L \varepsilon \\
& =\frac{C}{\varepsilon} \int_{0}^{2 \pi} \int_{\varepsilon}^{r_{\theta}} \int_{t}^{r_{\theta}}\left|\nabla f_{h}(t, \theta)\right| A(t, \theta) \mathrm{d} r \mathrm{~d} t \mathrm{~d} \theta+\mathcal{A}(S) L \varepsilon \\
& \leq \frac{C \operatorname{diam}(S)}{\varepsilon} \int_{0}^{2 \pi} \int_{\varepsilon}^{r_{\theta}}\left|\nabla f_{h}(t, \theta)\right| A(t, \theta) \mathrm{d} t \mathrm{~d} \theta+\mathcal{A}(S) L \varepsilon \\
& =\frac{C \operatorname{diam}(S)}{\varepsilon} \int_{S_{\varepsilon}}\left|\nabla f_{h}\right|+\mathcal{A}(S) L \varepsilon,
\end{aligned}
$$

where $\operatorname{diam}(S)$ is the diameter of $S$. Note that because the $\left(f_{h}\right)_{h>0}$ are $L$-Lipschitz and $f_{h}(b)=0$, we have

$$
\int_{S}\left|f_{h}\right|=\int_{S_{\varepsilon}}\left|f_{h}\right|+\int_{B(b, \varepsilon)}\left|f_{h}\right| \leq \int_{S_{\varepsilon}}\left|f_{h}\right|+\mathcal{A}(S) L \varepsilon .
$$

Therefore the estimate (5.17) yields

$$
\int_{S}\left|f_{h}\right| \leq \frac{C \operatorname{diam}(S)}{\varepsilon} \int_{S}\left|\nabla f_{h}\right|+2 \mathcal{A}(S) L \varepsilon
$$

In particular, for any $\varepsilon>0$,

$$
\limsup _{h \rightarrow 0} \int_{S}\left|f_{h}\right| \leq 2 \mathcal{A}(S) L \varepsilon
$$

This concludes the proof.

We can now establish the convergence of the minimizers:

\section{Proposition 5.4.}

$$
\left|\nabla_{S} u_{m, h}^{l}-\nabla_{S} u_{m}\right|_{L^{2}(S)}^{2}=\mathcal{O}\left(h^{\frac{1}{2}}\right) \quad \text { and } \quad\left|u_{m, h}^{l}-u_{m}\right|_{L^{1}(S)} \underset{h \rightarrow 0}{\longrightarrow} 0 .
$$

Proof. Consider $v=\frac{1}{2}\left(L u_{m, h}^{l}+u_{m}\right)$. Then, $v$ is admissible for problem (1.1), so $F(v) \geq F\left(u_{m}\right)$. Moreover, the following algebraic identity holds

$$
F(v)=\frac{1}{2} F\left(L u_{m, h}^{l}\right)+\frac{1}{2} F\left(u_{m}\right)-\frac{1}{4} \int_{S}\left|\nabla_{S} u_{m}-\nabla_{S} L u_{m, h}^{l}\right|^{2} .
$$

Therefore, we have

$$
\frac{1}{2} F\left(L u_{m, h}^{l}\right)-\frac{1}{2} F\left(u_{m}\right) \geq \frac{1}{4} \int_{S}\left|\nabla_{S} u_{m}-\nabla_{S} L u_{m, h}^{l}\right|^{2},
$$

which proves, together with Proposition 5.2, that

$$
\left|\nabla_{S} L u_{m, h}^{l}-\nabla_{S} u_{m}\right|_{L^{2}(S)}^{2}=\mathcal{O}\left(h^{\frac{1}{2}}\right) .
$$

In particular, as $S$ is compact, the gradient of the function $L u_{m, h}^{l}-u_{m}$ also goes to 0 in the $L^{1}(S)$ norm as $h \rightarrow 0$. Recall that the functions $\left(L u_{m, h}^{l}\right)_{h>0}$ and $u_{m}$ are uniformly Lipschitz, and $L u_{m, h}^{l}(b)=u_{m}(b)=0$, so we may apply Lemma 5.3 to the functions $\left(L u_{m, h}^{l}-u_{m}\right)_{h>0}$, to find that

$$
\left|L u_{m, h}^{l}-u_{m}\right|_{L^{1}(S)} \underset{h \rightarrow 0}{\longrightarrow} 0 .
$$

In the proof of Proposition 5.2, we showed that $L u_{m, h}^{l}=u_{m, h}^{l}(1+\mathcal{O}(h))$ (Eq. (5.14)). Together with (5.18) and (5.19), this concludes the proof. 
We just proved that the gradient of the lifted minimizers of the discrete problems (5.6) converge with an order at least $1 / 4$ to the gradient of the minimizer of problem (1.1).

\subsection{Convergence in measure of $E_{m, \lambda, h}$}

Let us recall that the set $E_{m, \lambda}$ is defined by

$$
E_{m, \lambda}=\left\{x \in S \backslash\{b\}:\left|\nabla_{S} u_{m}(x)\right|^{2} \leq 1-\frac{\lambda^{2}}{u_{m}^{2}(x)}\right\} .
$$

Proposition 5.5. For any $\lambda>0$, let us define

$$
E_{m, \lambda, h}:=\left\{x \in S \backslash\{b\}:\left|\nabla_{S} u_{m, h}^{l}(x)\right|^{2} \leq 1-\frac{\lambda^{2}}{\left(u_{m, h}^{l}\right)^{2}(x)}\right\} .
$$

For any $\varepsilon>0$ with $\varepsilon<\lambda / 2$, we have

$$
\left|E_{m, \lambda+\varepsilon} \backslash E_{m, \lambda, h}\right|=\mathcal{O}\left(h^{\frac{1}{4}}\right) \quad \text { and } \quad\left|E_{m, \lambda, h} \backslash E_{m, \lambda-\varepsilon}\right|=\mathcal{O}\left(h^{\frac{1}{4}}\right) .
$$

Proof. By definition of $E_{m, \lambda}$ and $E_{m, \lambda, h}$, we have

$$
E_{m, \lambda+\varepsilon} \backslash E_{m, \lambda, h} \subset\left\{\left|\nabla_{S} u_{m, h}^{l}\right|^{2}-\left|\nabla_{S} u_{m}\right|^{2}>\frac{(\lambda+\varepsilon)^{2}}{u_{m}^{2}}-\frac{\lambda^{2}}{\left(u_{m, h}^{l}\right)^{2}}\right\} .
$$

Therefore, on $E_{m, \lambda+\varepsilon} \backslash E_{m, \lambda, h}$, we have

$$
\begin{aligned}
\left|\nabla_{S} u_{m, h}^{l}\right|^{2}-\left|\nabla_{S} u_{m}\right|^{2} & >\frac{(\lambda+\varepsilon)^{2}-\lambda^{2}}{u_{m}^{2}}+\lambda^{2}\left(\frac{1}{u_{m}^{2}}-\frac{1}{\left(u_{m, h}^{l}\right)^{2}}\right) \\
& \geq \frac{2 \varepsilon \lambda+\varepsilon^{2}}{(\operatorname{diam}(S))^{2}}+\lambda^{2}\left(\frac{1}{u_{m}^{2}}-\frac{1}{\left(u_{m, h}^{l}\right)^{2}}\right),
\end{aligned}
$$

where $\operatorname{diam}(S)$ is the diameter of $S$. Recall that, by Proposition 5.4, $u_{m, h}^{l}$ converges to $u_{m}$ in $L^{1}(S)$ as $h$ goes to 0 . As the functions $u_{m}$ and $u_{m, h}^{l}$ are uniformly Lipschitz, this implies that $u_{m, h}^{l}$ converges to $u_{m}$ in $L^{\infty}(S)$. Moreover, by definition of $E_{m, \lambda}$, we also have $E_{m, \lambda+\varepsilon} \subset\left\{u_{m} \geq(\lambda+\varepsilon)\right\}$, so on $E_{m, \lambda+\varepsilon} \backslash E_{m, \lambda, h}$, the difference $\left(\frac{1}{u_{m}^{2}}-\frac{1}{\left(u_{m, h}^{l}\right)^{2}}\right)$ converges uniformly to 0 as $h$ goes to 0 . With (5.20), this implies that for $h$ small enough, we have on $E_{m, \lambda+\varepsilon} \backslash E_{m, \lambda, h}$,

$$
\left|\nabla_{S} u_{m, h}^{l}\right|^{2}-\left|\nabla_{S} u_{m}\right|^{2} \geq \frac{2 \varepsilon \lambda+\varepsilon^{2}}{2(\operatorname{diam}(S))^{2}} .
$$

In particular, setting $\eta:=\frac{2 \varepsilon \lambda+\varepsilon^{2}}{2(\operatorname{diam}(S))^{2}}$ and using Proposition 5.4, we obtain

$$
\begin{aligned}
\left|E_{m, \lambda+\varepsilon} \backslash E_{m, \lambda, h}\right| & \leq\left|\left\{\left|\nabla_{S} u_{m, h}^{l}\right|^{2}-\left|\nabla_{S} u_{m}\right|^{2}>\eta\right\}\right| \\
& \leq\left.\frac{1}{\eta} \int_{S}|| \nabla_{S} u_{m, h}^{l}\right|^{2}-\left|\nabla_{S} u_{m}\right|^{2} \mid \\
& =\mathcal{O}\left(h^{\frac{1}{4}}\right) .
\end{aligned}
$$

This concludes the proof of the first estimate. The other estimate is proved by the same method. 


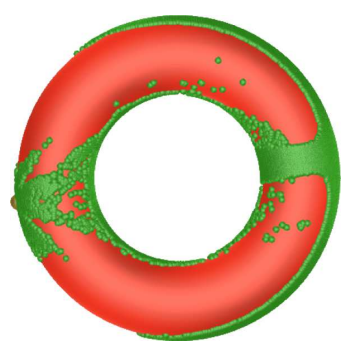

FiguRE 2. Artifacts using low order approximation.

Remark 5.6. The function $u_{m}$ is generically suspected to be no more than $\mathcal{C}^{1,1}$ regular. This important difficulty makes the extension of the previous convergence analysis to higher order elements not straightforward. However, based on our numerical experiments (see the following paragraph), we believe that a $(k, r)$ approximation improves the order of convergence without being able to provide a complete analysis of this order due to this lack of regularity.

Sections 3-5 together justify the claim that the set $E_{m, \lambda, h}$ is a good approximation of the cut locus of $b$ in $S$, if $m$ is big enough and $\lambda$ and $h$ are small enough. We tried to estimate numerically the order of convergence of our approximation. Unfortunately, the precision of our simulations was not enough to determine if the expected order of convergence $1 / 4$ is optimal or not.

\section{NumericAl illustrations}

\subsection{Cut locus approximation}

We established the convergence of the minimizers of problem (5.6) when $h$ tends to 0 . For a fixed $h>0$, this convex discrete problem is of quadratic type with an infinite number of conic pointwise constraints. By the way, it is important to observe that for $k=r=1$, the gradient pointwise bounds for a function of $\mathbb{P}^{1}$ is equivalent to a single discrete conic constraint on every triangle with respect to the degrees of freedom of $\mathbb{P}^{1}\left(\mathcal{T}_{h}\right)$. In this simplified context, we observed in our experiments that using $\mathbb{P}^{1}$ elements may lead to approximated cut loci with some tiny artificial connected components (see Fig. 2). Observe that these artifacts do not contradict our convergence estimates in Proposition 5.5. Motivated by this lack of precision, we use in all following illustrations elements of order $r>1$.

For the general case $r>1$, the bound constraint on the gradient cannot be easily reduced to a finite set of discrete constraints. In our computations, we approximated the constraint $\left|\nabla_{S_{h}^{k}} u\right|_{L^{\infty}\left(S_{h}^{k}\right)} \leq 1$ by forcing the inequality only on a finite number of points of the mesh. In practice, we imposed these constraints on $g$ Gauss quadrature points on every triangle of $\mathcal{T}_{h}$.

We illustrate in Figures 3-6 the approximation of the cut locus provided by our approach. These computations have been carried out on meshes of approximately $10^{5}$ triangles for $k=2$ and $r=3$ using a high precision quadrature formula associated to 17 Gauss points on every element of the mesh. Moreover, for $r=3$, we imposed the conic gradient constraints on the $g=9$ Gauss points of every triangle. In order to solve the resulting linear conic constrained quadratic optimization problem, we used the JuMP modeling language and the finite elements library Getfem $++[10,17]$ combined with the MOSEK optimization solver [2]. For such a precision, the optimization solver identified a solution in less than one hour on a standard computer.

Observe that our approximations of cut loci provide sets with a number of handles equal to twice the genus of the supporting surfaces. This fact is in agreement with Proposition 3.2 since the cut locus has he same homotopy group as the surface (see [6] for instance). 

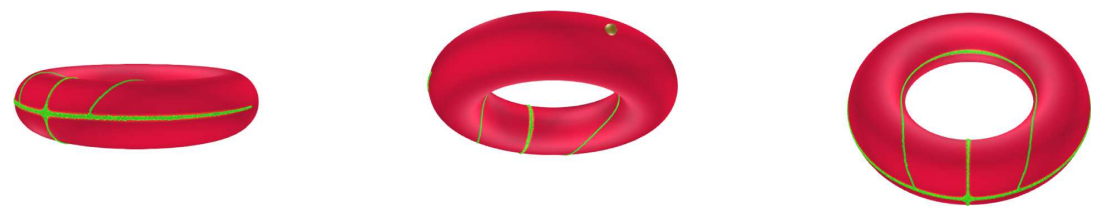

FiguRE 3. Three different views of the approximation of a cut locus on a standard torus.
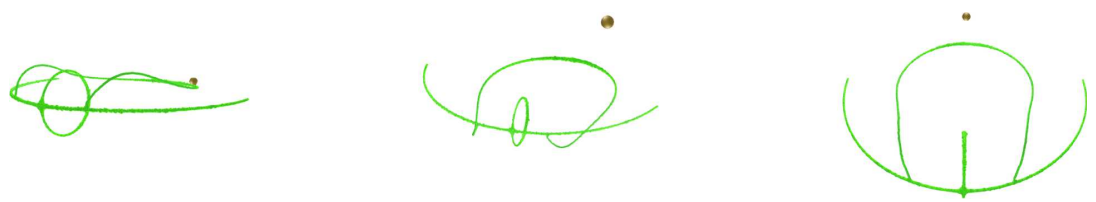

FiguRE 4. Three different views of the approximation of a cut locus on a standard torus, without representing the surface.
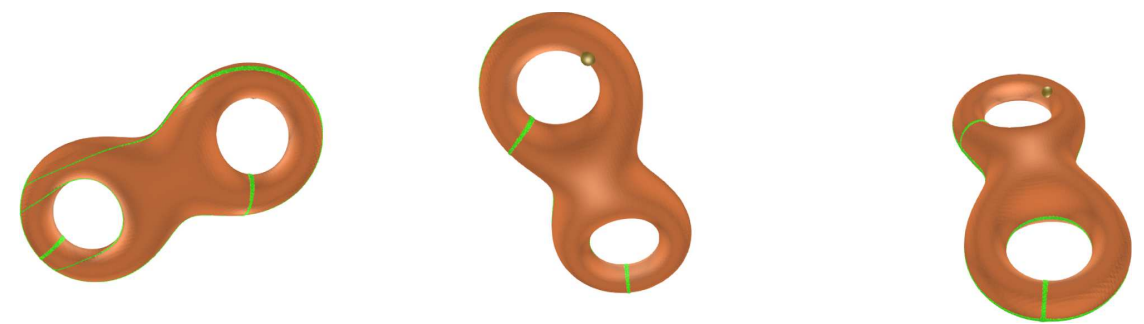

Figure 5. Three different views of the approximation of a cut locus on a torus of genus 2.
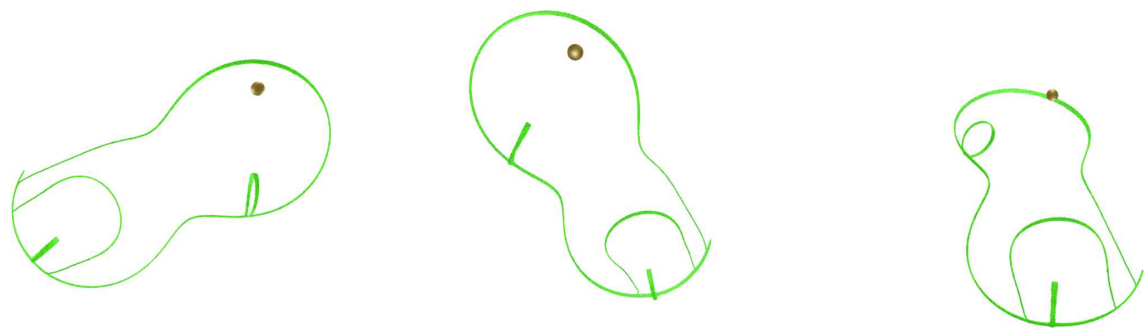

FiguRE 6. Three different views of the approximation of a cut locus on a torus of genus 2, without representing the surface.

\subsection{Approximation of the boundary of Voronoi cells}

All previous theoretical results still hold if we replace the source point $b$ by any compact subset of the surface $S$. For instance, if $b$ is replaced by a set of points, the singular set of the distance function can be decomposed as the union of the boundary of Voronoi cells and the cut loci of every point intersected with its Voronoi cell. As a consequence, if the distribution of source points is homogeneous enough, that is every Voronoi cell is small enough, the singular part of the distance function will be exactly equal to the boundary of the Voronoi cells. We illustrate this remark in the following experiments. We used exactly the same framework as in previous sections and just replaced the pointwise condition at $b$ with the analogous pointwise Dirichlet conditions at every source 

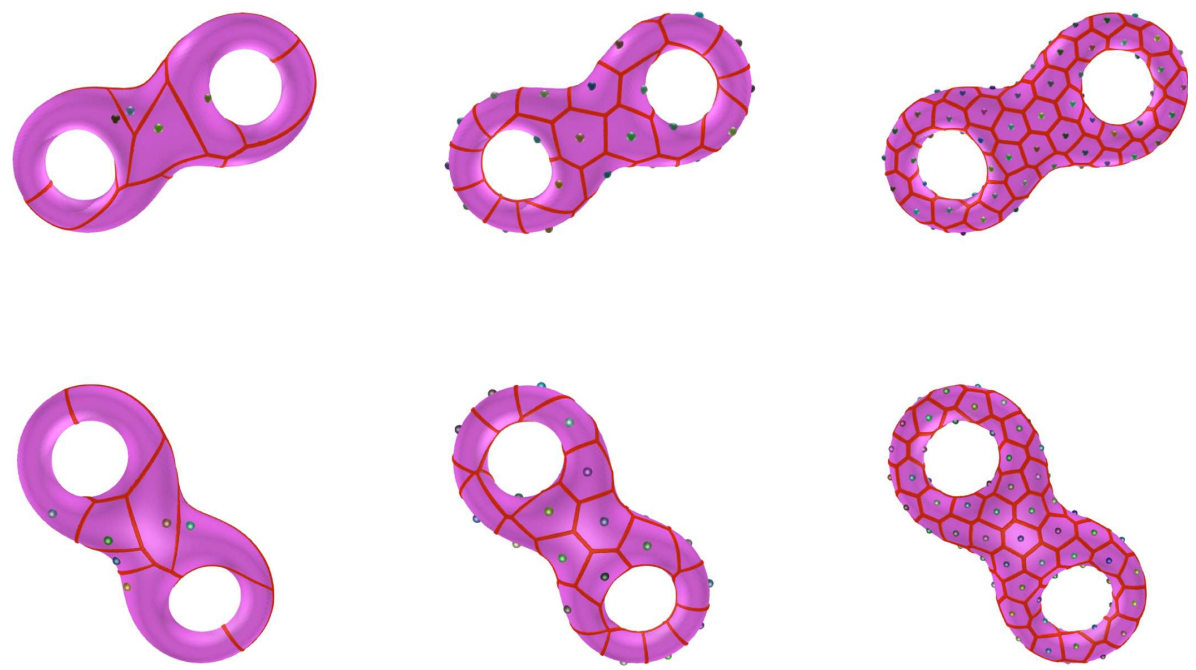

Figure 7. Approximation of the Voronoi cells on a torus of genus 2 of 10, 30 and 100 points. Every column represents two different views.
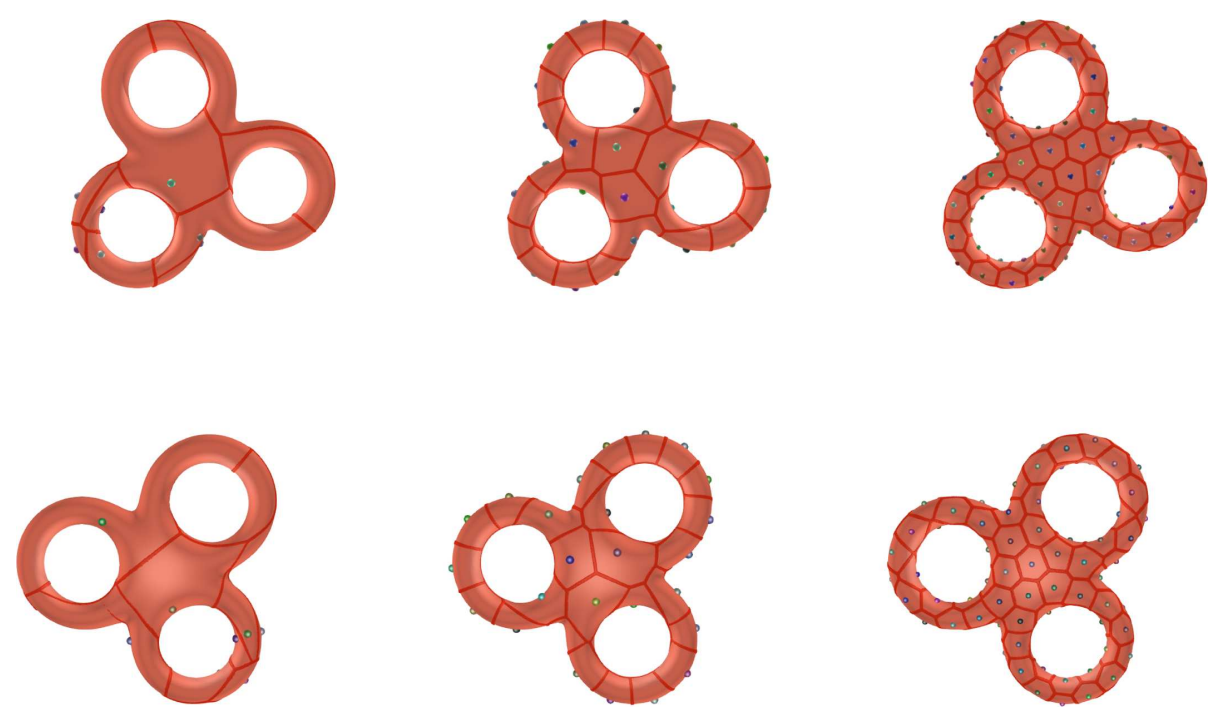

Figure 8. Approximation of the Voronoi cells on a torus of genus 3 of 10, 30 and 100 points. Every column represents two different views.

point. Figures 7 and 8 represent the Voronoi diagrams obtained with 10, 30 and 100 points for surfaces of genus 2 and 3. The computational complexity is exactly of the same order as with a single source point.

Acknowledgements. The three authors were partially supported by Agence Nationale de la Recherche (ANR) with the projects GeoSpec (LabEx PERSYVAL-Lab, ANR-11-LABX-0025-01), CoMeDiC (ANR-15-CE40-0006) and ShapO (ANR-18-CE40-0013). The third author is supported by the European Research Council (ERC), under the European Union's Horizon 2020 research and innovation programme, through the project ERC VAREG - Variational approach to the regularity of the free boundaries (grant agreement No. 853404). 


\section{REFERENCES}

[1] P. Albano, On the stability of the cut locus. Nonlinear Anal. Theory Methods App. 136 (2016) 51-61.

[2] E.D. Andersen and K.D. Andersen, The mosek interior point optimizer for linear programming: an implementation of the homogeneous algorithm. In: High Performance Optimization. Springer (2000) 197-232.

[3] D. Attali and A. Montanvert, Modeling noise for a better simplification of skeletons. In: Proceedings of 3rd IEEE International Conference on Image Processing. Vol. 3. IEEE (1996) 13-16.

[4] B. Bonnard, O. Cots and L. Jassionnesse, Geometric and numerical techniques to compute conjugate and cut loci on Riemannian surfaces. In: Geometric Control Theory and Sub-Riemannian Geometry. Springer (2014) 53-72.

[5] M.A. Buchner, Simplicial structure of the real analytic cut locus. Proc. Am. Math. Soc. 64 (1977) 118-121.

[6] I. Chavel, Riemannian Geometry: A Modern Introduction. Vol. 98. Cambridge University Press (2006).

[7] F. Chazal and A. Lieutier, The " $\lambda$-medial axis". Graphical Models 67 (2005) 304-331.

[8] A. Demlow, Higher-order finite element methods and pointwise error estimates for elliptic problems on surfaces. SIAM J. Numer. Anal. 47 (2009) 805-827.

[9] T.K. Dey and K. Li, Cut locus and topology from surface point data. In: Proceedings of the Twenty-Fifth Annual Symposium on Computational Geometry. ACM (2009) 125-134.

[10] I. Dunning, J. Huchette and M. Lubin, Jump: a modeling language for mathematical optimization. SIAM Rev. 59 (2017) $295-320$.

[11] G. Dziuk and C.M. Elliott, Finite element methods for surface PDEs. Acta Numer. 22 (2013) 289-396.

[12] F. Générau, E. Oudet and B. Velichkov, Cut locus on compact manifolds and uniform semiconcavity estimates for a variational inequality. Preprint arXiv:2006.07222 [math] (2020).

[13] J.-I. Itoh and R. Sinclair, Thaw: a tool for approximating cut loci on a triangulation of a surface. Exp. Math. 13 (2004) $309-325$.

[14] M.K. Misztal, J.A. Bærentzen, F. Anton and S. Markvorsen, Cut locus construction using deformable simplicial complexes. In: 2011 Eighth International Symposium on Voronoi Diagrams in Science and Engineering. IEEE (2011) $134-141$.

[15] S.B. Myers, Connections between differential geometry and topology II. Closed surfaces. Duke Math. J. 2 (1936) 95-102.

[16] A. Petrunin, Semiconcave functions in Alexandrov's geometry. Surv. Differ. Geom. 11 (2006) 137-202.

[17] Y. Renard and J. Pommier, Getfem++. An open source generic C++ library for finite element methods (http://home.gna. org/getfem) (2006).

[18] R. Sinclair and M. Tanaka, Loki: software for computing cut loci. Exp. Math. 11 (2002) 1-25.

\section{Subscribe to Open (S20) A fair and sustainable open access model}

This journal is currently published in open access under a Subscribe-to-Open model (S2O). S2O is a transformative model that aims to move subscription journals to open access. Open access is the free, immediate, online availability of research articles combined with the rights to use these articles fully in the digital environment. We are thankful to our subscribers and sponsors for making it possible to publish this journal in open access, free of charge for authors.

\section{Please help to maintain this journal in open access!}

Check that your library subscribes to the journal, or make a personal donation to the S2O programme, by contacting subscribers@edpsciences.org

More information, including a list of sponsors and a financial transparency report, available at: https://www. edpsciences.org/en/maths-s2o-programme 